\title{
Liegt in männlichen Lehrkräften der Schlüssel zum Schulerfolg der Jungen? \\ Befunde aus Schülerbefragungen
}

\author{
Dirk Baier, Thomas Mößle, Christian Pfeiffer
}

\section{Einleitung}

Seit einiger Zeit wird über den geringeren Schulerfolg männlicher Kinder und Jugendlicher im Vergleich zu weiblichen Kindern und Jugendlichen diskutiert. Dieser ausbleibende Schulerfolg schlägt sich in höheren Quoten an Schulabbrechern bei männlichen Jugendlichen ebenso nieder wie in einer rückläufigen Gymnasialquote. Zudem zeigen sich in Kompetenztests und Schulnoten Geschlechtsunterschiede zugunsten der Mädchen (vgl. u. a. Quenzel \& Hurrelmann, 2010). Die Frage nach den Ursachen dieser Entwicklung ist bislang nur wenig untersucht. In der öffentlichen Diskussion findet sich in erster Linie die Auffassung, dass die Leistungskrise der Jungen mit einer Feminisierung des Lehrerberufs in Zusammenhang steht. Neugebauer (2011, S. 236) zeigt hiermit übereinstimmend, dass zwischen 1953 und 2007 der Anteil an Lehrerinnen an allgemeinbildenden Schulen von 36 auf $69 \%$ gestiegen ist. Besonders deutliche Anstiege hat es dabei einerseits bis Ende der 1970er-Jahre geben, andererseits aber auch seit Anfang der 1990er-Jahre. Gerade seit Anfang der 1990er-Jahre beginnt sich die Schere im Schulerfolg bei Jungen und Mädchen zu öffnen.

Die These der Feminisierung des Lehrerberufs besagt, dass Jungen ihr Leistungspotenzial nicht optimal abrufen können, wenn sie von Frauen unterrichtet werden. Je mehr Frauen den Lehrerberuf ausüben, desto mehr Jungen sind hiervon betroffen. Dass es auf Aggregatebene einen entsprechenden Zusammenhang zu geben scheint, bedeutet jedoch nicht, dass dieser Zusammenhang tatsächlich empirisch als belegt gelten kann. Hierzu ist die Auswertung von Individualdaten notwendig. In diesem Beitrag wird unter Rekurs auf verschiedene Schülerbefragungen des Kriminologischen Forschungsinstituts Niedersachsen (KFN) die These geprüft, dass Jungen, die von Lehrerinnen unterrichtet werden, schlechtere Leistungen erzielen als Mädchen. Zuvor soll der Forschungstand zu dieser These vorgestellt werden, da es einige wenige Studien aus Deutschland gibt, die ge- 
prüft haben, inwieweit sich das Geschlecht der Lehrkraft auf die Schulleistungen auswirkt.

\section{Bisherige Befunde}

Dass das Geschlecht der Lehrkraft mit dem Schulerfolg in Zusammenhang stehen könnte, lässt sich in zweierlei Weise begründen. Eine erste Argumentation besagt, dass die Identifikation mit einer Lehrkraft die schulische Bindung stärkt und dazu motiviert, sich in der Schule anzustrengen. Durch die Zunahme des Anteils an Frauen im Lehrpersonal mangelt es „Jungen an Rollenmodellen bzw. Vergleichspersonen [...], an denen sie ihre Fähigkeitsselbsteinschätzungen ausrichten und aus denen sie ihre Motivation beziehen können“ (Diefenbach, 2010, S. 261). Das Augenmerk richtet sich in dieser Argumentation damit insbesondere auf die Schüler.

Die zweite Argumentation richtet sich auf die Lehrkräfte. In der Schule wird ein bestimmtes Verhalten erwartet: Die Schüler sollen lernbereit sein und gewaltfrei miteinander umgehen. Ein entsprechendes Verhalten wird als entscheidend für den Schulerfolg angesehen. Schülern, die sich nicht in dieser Weise verhalten, wird die Fähigkeit zum schulischen Erfolg eher abgesprochen. „Möglicherweise werden Lehrerinnen durch solches Verhalten stärker, irritiert' als männliche Lehrer, wenn sie als Maßstab die eigene geschlechtsspezifische Sozialisation heranziehen" (Neugebauer, 2011, S. 241). Weibliche Lehrkräfte bringen den Jungen, die diese Verhaltensweisen häufiger ausüben, weniger Verständnis entgegen, trauen ihnen weniger $\mathrm{zu}$ und empfehlen sie dann auch seltener für eine höhere Bildungslaufbahn. In diesem Sinne würden die weiblichen Lehrkräfte Jungen nicht aufgrund der tatsächlichen Leistungen, sondern aufgrund sekundärer Kriterien bewerten.

Anhand der vorliegenden empirischen Befunde kann allerdings in Zweifel gezogen werden, dass männliche Schüler besonders gut bei männlichen Lehrkräften lernen bzw. dass weibliche Lehrkräfte männliche Schüler benachteiligen würden. So berichten Baier und Pfeiffer (2011) auf Basis einer Befragung unter Grundschülern der vierten Jahrgangsstufe, dass das Geschlecht der Lehrkraft weder einen Einfluss auf die Notenvergabe noch auf die Vergabe von Laufbahnempfehlungen hat. Zugleich finden sich keine Hinweise darauf, dass Jungen in dieser Hinsicht von weiblichen Lehrkräften benachteiligt würden. „Männliche Schüler scheinen demnach nicht von einer männlichen Lehrkraft zu profitieren; es ist eher das Gegenteil der Fall“" (S. 272). 
Die Studien von Neugebauer, Helbig und Landmann (2010) und Neugebauer (2011) bestätigen den Befund, dass das Lehrergeschlecht weitestgehend irrelevant für den Schulerfolg von männlichen Schülern ist. Dabei untersuchen Neugebauer et al. (2010) den Zusammenhang mit Kompetenzwerten. Weder Jungen noch Mädchen profitieren in ihrer Kompetenzentwicklung von einer Lehrkraft desselben Geschlechts. Alle Kinder erreichen bei weiblichen Lehrkräften etwas bessere Kompetenzwerte. In einer weiteren Analyse bezieht Neugebauer (2011) zusätzlich die gymnasialen Laufbahnempfehlungen ein. Zentrale Erkenntnis ist, dass ,Jungen [...] nicht von weiblichen Lehrkräften benachteiligt [werden; d. A.], wenn es um eine gymnasiale Laufbahnempfehlung geht" (Neugebauer, 2011, S. 255). Nachteilig an dieser Studie ist allerdings, dass nicht das Geschlecht der die Laufbahnempfehlung erteilenden Lehrkraft in den Auswertungen berücksichtig werden kann, sondern nur die Geschlechterzusammensetzung der Lehrerschaft der Schule.

Diese Einschränkung gilt ebenfalls für die Studie von Helbig (2010). Unter Bezugnahme auf Leistungstests und Schulnoten unter Berliner Grundschülern wird festgestellt, dass Jungen weitestgehend unbeeinflusst vom Lehrerinnenanteil einer Schule sind. Es findet sich aber ein schwacher Effekt derart, dass Jungen in Schulen mit einem hohen Anteil an Lehrerinnen schlechter im Fach Mathematik benotet werden, was zur Folge hat, dass sie letztlich auch etwas seltener auf das Gymnasium wechseln. Zudem findet sich ein positiver Einfluss des Lehrerinnenanteils auf Mädchen: Mit steigendem Anteil steigt auch die Lesekompetenz der weiblichen Schüler.

In einer weiteren Studie analysiert Helbig (2010a) den Einfluss des Lehrergeschlechts auf die Kompetenzentwicklung anhand einer 21 Länder (inkl. Deutschland) umfassenden Stichprobe von Kindern der vierten Klasse. Er stellt heraus, „dass Jungen bei ihrer Kompetenzentwicklung nicht durch den Unterricht bei einer männlichen Lehrkraft profitieren“ (Helbig, 2010a, S. 284). Gleichzeitig profitieren auch die Mädchen nicht vom Unterricht bei einer weiblichen Lehrkraft.

Auch Hadjar und Lupatsch (2011) gehen anhand einer schweizerischen Jugendstichprobe der Frage nach dem Einfluss der Lehrperson auf den geschlechtsspezifischen Schulerfolg nach. Übereinstimmend mit den Befunden der bereits genannten Studien konstatieren sie, „dass hinter den Geschlechterunterschieden im Schulerfolg keine einseitigen bewussten oder unbewussten Diskriminierungen durch Lehrerinnen stehen" (S. 91). Die Auswertungen anhand von Strukturgleichungsmodellen bieten zugleich verschiedene weitergehende Erkenntnisse. So zeigt sich, dass das 
Lehrergeschlecht indirekt von (geringer) Bedeutsamkeit für den Schulerfolg ist, insofern es die „Fächerassoziation“ beeinflusst, d. h. die Wahrnehmung, ob ein Fach als eher männlich oder eher weiblich von den Schülern eingestuft wird. Diese Assoziation beeinflusst wiederum das Interesse an einem Fach und dieses Interesse hat Auswirkungen auf den Schulerfolg. Männliche Deutschlehrer haben bspw. zur Folge, dass Jungen Deutsch seltener als eher weibliches Fach einstufen, was ihr Interesse und letztlich ihren Schulerfolg erhöht. Ein anderer wichtiger Befund ist, dass die Schulentfremdung starken Einfluss auf das Fachinteresse hat. Die Schulentfremdung beinhaltet $u$. a. eine negative Einstellung zur Schule; bei Jungen ist die Entfremdung stärker ausgeprägt als bei Mädchen. Die Entfremdung ist wiederum abhängig von der sozialen Herkunft (eine niedrige Sozialschicht erhöht die Entfremdung) und der Unterstützung durch Lehrpersonen in der Schule.

Die vorhandenen empirischen Befunde können damit die These, dass Jungen in ihrem Schulerfolg durch weibliche Lehrkräfte gebremst werden, nicht bestätigen. Stattdessen gibt es Hinweise darauf, dass Jungen durch weibliche Lehrkräfte sogar etwas stärker gefördert werden. Aber auch dabei gilt, dass die Befunde nicht einheitlich sind und alles in allem eher schwach ausfallen. Wenn die Befundlage aber derart einheitlich ist, welche Gründe sprechen dann dafür, diese These ein weiteres Mal einer empirischen Prüfung auszusetzen? Drei Gründe sind aus unserer Sicht hierfür anzuführen:

1. Im Rahmen verschiedener Schülerbefragungen des KFN wurden Daten zu den Schulleistungen und dem Geschlecht der Lehrkräfte erhoben. Hierunter befinden sich auch Befragungen, die als deutschlandweit repräsentativ gelten können und die eine sehr hohe Befragtenanzahl aufweisen. Das Geschlecht der Lehrkraft wurde in diesen Befragungen direkt erfasst, so dass nicht auf eine Proxy-Variable wie die Geschlechterzusammensetzung des Lehrerkollegiums zurückgegriffen werden muss. Hinzu kommt, dass gleichzeitig verschiedene Altersgruppen betrachtet werden können. Die bisherigen Studien konzentrieren sich meist auf die Grundschule, weil in den meisten Bundesländern danach die Zuweisung zu weiteren Schulen erfolgt - mit den diesen Übergang strukturierenden Laufbahnempfehlungen. In den Schülerbefragungen wurden neben der vierten aber auch die neunten Jahrgangsstufen untersucht. Möglicherweise spielen hier Prozesse der Identifikation mit der gleichgeschlechtlichen Lehrkaft oder der Be- 
nachteiligung von Schülern durch gegengeschlechtliche Lehrkräfte eine größere Rolle.

2. Die bisherigen Studien konzentrieren sich darauf, das Lehrer-SchülerVerhältnis im Wesentlichen von der Seite der Lehrkräfte zu betrachten. Es wird gefragt, ob Lehrkräfte die Schüler aufgrund der Geschlechtszugehörigkeit unterschiedlich bewerten oder nicht. Wenn es solche Prozesse geben würde, dann wäre denkbar, dass sich diese auch in der umgekehrten Richtung zeigen, d. h. in der Bewertung der Lehrer durch die Schüler. Die Berücksichtung der Bewertungen der Lehrer durch die Schüler würde es zudem möglich machen, die These zu prüfen, ob Schüler sich tatsächlich stärker mit Lehrkräften des eigenen Geschlechts identifizieren als mit Lehrkräften des anderen Geschlechts. Aus diesem Grund werden in den nachfolgenden Analysen auch die Bewertungen der Lehrkräfte durch die Schüler berücksichtigt.

3. Die These, dass die Leistungskrise der Jungen durch die Feminisierung des Lehrerberufs bedingt ist, ist eine der wenigen, die zur Erklärung dieser Entwicklung in den letzten 20 Jahren formuliert wurde. Die Gegenhypothese, die verschiedentlich empirisch geprüft wurde, geht davon aus, dass die Leistungskrise auf eine geschlechtsspezifische Veränderung des Freizeitverhaltens zurückzuführen ist (vgl. Baier \& Pfeiffer, 2011, S. 263ff). Einerseits belegen verschiedene Studien, dass der Medienkonsum in den letzten Jahren im Kindes- und Jugendalter deutlich zugenommen hat (Baier, 2008; Ribeaud \& Eisner, 2009). Andererseits bestätigen zahlreiche Befragungen, dass Jungen verschiedenen medienbezogenen Tätigkeiten zeitlich intensiver und in inhaltlich problematischerer Weise nachgehen als Mädchen. So widmen sich Jungen bedeutend länger dem Computerspielen und sie kommen auch deutlich häufiger als Mädchen mit altersunangemessenen Filmen und Computerspielen in Kontakt (Baier, Pfeiffer, Rabold, Simonson \& Kappes, 2010). Der Anteil computerspielabhängiger Jungen übersteigt den der Mädchen um das zehnfache (Rehbein, Kleimann \& Mößle, 2010). Weiterhin bestätigen mittlerweile verschiedene Längsschnittstudien, dass ein zeitlich ausgedehnter und inhaltlich problematischer Medienkonsum negativ auf die Schulleistungen wirkt (Ennemoser, 2003; Baier \& Pfeiffer, 2011). Anliegen dieses Beitrag ist es daher, zusätzlich zur These des Einflusses des Lehrergeschlechts zu prüfen, inwieweit die These bestätigt werden kann, dass Medienumgangsweisen mit dem Schulerfolg in Zusammenhang ste- 
hen und insofern eine Erklärung für das diesbezügliche Auseinanderdriften der Geschlechter in den letzten Jahrzehnten darstellen.

Um den Einfluss dieser beiden Faktoren (Lehrergeschlecht, Medienkonsum) nicht zu überschätzen, sollen gleichzeitig weitere Determinanten der Schulleistungen in die Betrachtung einbezogen werden. Hinzuweisen ist an dieser Stelle, dass die Daten nur erlauben, die Schulnoten sowie die Lehrkräfteeinschätzungen zu untersuchen. Informationen zu Laufbahnempfehlungen, Kompetenzwerten, tatsächlichen Schulübertritten o. ä. stehen uns nicht zur Verfügung. Wird der Forschungstand zu den Determinanten der Schulleistungen betrachtet, so lassen sich mindestens folgende Faktoren als relevant benennen (vgl. u. a. Mößle, 2012):

- Intelligenz

- Bildungsniveau bzw. Schichtzugehörigkeit

- Elterliches Unterstützungsverhalten

- Schulbindung und Lernbereitschaft

- Traditionelle Geschlechtsrollenorientierung

- Lernförderliche Freizeitaktivitäten wie Lesen oder Musizieren

- Schulbezogene Verhaltensauffälligkeiten

- Persönlichkeitseigenschaften (z. B. Selbstkontrolle)

Nicht alle diese Faktoren wurden im Rahmen der Schülerbefragungen erfasst, was dem Umstand geschuldet ist, dass diese Befragungen primär der Untersuchung des delinquenten Verhaltens von Kindern und Jugendlichen dienen. Zugleich ist es nicht notwendig, alle diese Faktoren in Erklärungsmodellen zu berücksichtigen, da nicht der Anspruch besteht, eine umfassende Analyse zu den Einflussfaktoren von Schulleistungen zu präsentieren.

\section{Ergebnisse}

3.1 Befunde einer Befragung von Schülern der 4. Jahrgangsstufe

In den Jahren 2007 und 2008 hat das KFN deutschlandweit 7,844 Kinder der vierten Jahrgangsstufe befragt (vgl. Baier et al., 2010, S. 258ff). Hierzu wurden 30 Landkreise bzw. kreisfreie Städte in insgesamt zwölf Bundesländern ausgewählt. Die Befragungen wurden in Schulklassen unter Aufsicht eines Testleiters während des Schulunterrichts durchgeführt. Ins- 
gesamt wurden 473 Klassen erreicht; die Rücklaufquote beträgt 53.9\%. Details zur Stichprobe können bei Baier et al. (2010, S. 261ff) nachgelesen werden.

In einem Teil des Fragebogens wurden die Schulnoten (Deutsch, Mathematik, Sport) des letzten Zeugnisses entsprechend der herkömmlichen Metrik. Ergebnisse aus Studien, die die Angaben der Kinder und der Lehrkräfte zu den Schulnoten vergleichen, kommen zu dem Schluss, dass Kinder ihre Schulnoten in sehr verlässlichem Maß berichten, sich also zum Großteil nicht besser machen, als sie wirklich sind (vgl. Baier, Pfeiffer, Windzio \& Rabold, 2006, S. 89ff). Aus diesem Grund wurde darauf verzichtet, die Schulnoten zusätzlich von den Lehrkräften mitteilen zu lassen.

Die Kinder wurden darüber hinaus gebeten, das Geschlecht des Klassenlehrers mitzuteilen sowie den Klassenlehrer mittels Schulnoten entlang von drei Fragen einzuschätzen: 1. Wie spannend ist der Unterricht? 2. Wie gerecht ist er/sie dir gegenüber? 3. Wie nett ist er/sie dir gegenüber? Die Einschätzungen zu allen drei Dimensionen korrelieren ausreichend hoch miteinander, weshalb sie zu einer Skala „Bewertung Klassenlehrkraft“ zusammengefasst werden können (Cronbachs Alpha $=.83, \mathrm{~N}=7,562$ ).

Ein Problem der Daten ist, dass erstens nicht zu allen Klassen Angaben zum Geschlecht der Lehrkräfte vorliegen und dass zweitens z. T. von den Schülern einer Klasse unterschiedliche Geschlechter der unterrichtenden Lehrkräfte mitgeteilt worden sind. Dies ist plausibel, da bspw. im Fach Sport sowohl weibliche als auch männliche Lehrkräfte in einer Klasse unterrichten können. Möglich ist auch, dass einzelne Schüler einer Klasse im Rahmen von Förderunterricht o. ä. primär von anderen Lehrkräften unterrichtet werden, die dann ein anderes Geschlecht haben können. Wir haben uns daher dazu entschieden, nur jene Klassen in die Auswertungen einzubeziehen, in denen alle befragten Schüler dasselbe Lehrergeschlecht berichtet haben. Dies erscheint deshalb sinnvoll, weil nur in diesen Klassen untersucht werden kann, ob Lehrer eines bestimmten Geschlechts Schülerinnen und Schüler unterschiedlich behandeln. Diese Entscheidung führt allerdings zu einer verringerten Fallzahl. Statt Schüler aus 473 Klassen können bei der Analyse der Deutschnote nur Schüler aus 410 Klassen, bei der Mathematiknote Schüler aus 392 Klassen, bei der Sportnote aus 342 Klassen und bei der Analyse der Klassenlehrerbewertung nur Schüler aus 468 Klassen einbezogen werden. 
Tabelle 1. Noten und Klassenlehrkraftbewertungen nach Geschlecht des Schülers und der Lehrkraft (Mittelwerte).

\begin{tabular}{|c|c|c|c|c|c|}
\hline $\begin{array}{l}\text { Geschlecht } \\
\text { Befragter }\end{array}$ & $\begin{array}{l}\text { Geschlecht } \\
\text { Lehrkraft }\end{array}$ & $\begin{array}{c}\text { Deutschnote } \\
(N=6,438)\end{array}$ & $\begin{array}{l}\text { Mathematiknote } \\
\qquad(N=6,134)\end{array}$ & $\begin{array}{l}\text { Sportnote } \\
(N=5,304)\end{array}$ & $\begin{array}{c}\text { Bewertung } \\
\text { Klassenlehr- } \\
\text { kraft } \\
(\mathrm{N}=\mathbf{7 , 6 4 3 )}\end{array}$ \\
\hline \multirow[t]{2}{*}{ Männlich } & Weiblich & 2.60 & 2.35 & 1.87 & 2.26 \\
\hline & Männlich & 2.53 & 2.43 & 1.92 & 2.35 \\
\hline \multirow[t]{2}{*}{ Weiblich } & Weiblich & 2.34 & 2.53 & 1.93 & 1.98 \\
\hline & Männlich & 2.43 & 2.67 & 1.92 & 2.14 \\
\hline \multirow{3}{*}{ F-Werte } & & $\mathrm{F}_{\mathrm{BG}}=11.27^{* *}$ & $\mathrm{~F}_{\mathrm{BG}}=29.83^{* * *}$ & $\mathrm{~F}_{\mathrm{BG}}=1.28$ & $\mathrm{~F}_{\mathrm{BG}}=37.71^{* * *}$ \\
\hline & & $F_{L G}=0.04$ & $\mathrm{~F}_{\mathrm{LG}}=9.03^{* *}$ & $F_{L G}=0.46$ & $F_{L G}=9.67^{* *}$ \\
\hline & & $\mathrm{F}_{\mathrm{I}=2.08}$ & $\mathrm{~F}_{\mathrm{I}}=0.57$ & $\mathrm{~F}_{\mathrm{I}=0.74}$ & $\mathrm{~F}_{\mathrm{I}=0.64}$ \\
\hline
\end{tabular}

Anmerkung. $\mathrm{F}_{\mathrm{BG}}=\mathrm{F}$-Wert Befragtengeschlecht, $\mathrm{F}_{\mathrm{LG}}=\mathrm{F}-$ Wert Lehrergeschlecht,

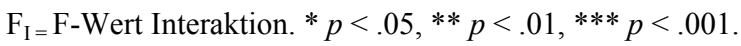

In Tabelle 1 sind die Mittelwerte der Noten sowie der Bewertung nach dem Geschlecht des Schülers und der Lehrkraft abgebildet, wobei die Mittelwertsunterschiede mittels zweifaktorieller Varianzanalysen auf Signifikanz geprüft wurden. Für die Deutsch- und die Mathematiknote ergibt sich jeweils ein signifikanter Unterschied nach dem Geschlecht des Kindes. Weibliche Grundschüler erzielen bessere Deutsch-, zugleich aber schlechtere Mathematiknoten. Für die Mathematiknote findet sich darüber hinaus ein signifikanter Lehrkraftgeschlechteffekt: Männliche Lehrkräfte vergeben schlechtere Noten als weibliche Lehrkräfte. Entscheidend ist, dass es bei keiner der Schulnoten einen signifikanten Interaktionseffekt gibt; d. h. es lässt sich nicht nachweisen, dass männliche Schüler von männlichen Lehrkräften profitieren oder weibliche Schüler von weiblichen Lehrkräften. Ein solcher Effekt deutet sich nur bei der Deutschnote an: Die besten Deutschnoten erhalten Schülerinnen bei Lehrerinnen, die schlechtesten Schüler bei Lehrerinnen. Männliche Schüler, die von Lehrern unterrichtet werden, haben geringfügig bessere Noten als männliche Schüler bei Lehrerinnen. Diese Unterschiede werden aber nicht als signifikant ausgewiesen, und dies bei einer Fallzahl von fast 6,500 Schülern. 
Tabelle 2. Klassenlehrkraftbewertungen nach Geschlecht des Schülers und der Lehrkraft (Mittelwerte).

\begin{tabular}{|c|c|c|c|c|}
\hline $\begin{array}{l}\text { Geschlecht } \\
\text { Befragter }\end{array}$ & $\begin{array}{l}\text { Geschlecht } \\
\text { Klassenlehrkraft }\end{array}$ & $\begin{array}{c}\text { Spannender } \\
\text { Unterricht } \\
(\mathrm{N}=7,603)\end{array}$ & $\begin{array}{c}\text { Gerechtigkeit } \\
(\mathbf{N}=7,584)\end{array}$ & $\begin{array}{l}\text { Nettigkeit } \\
(N=7,585)\end{array}$ \\
\hline \multirow[t]{2}{*}{ Männlich } & Weiblich & 2.56 & 2.26 & 1.96 \\
\hline & Männlich & 2.52 & 2.42 & 2.11 \\
\hline \multirow[t]{2}{*}{ Weiblich } & Weiblich & 2.35 & 1.94 & 1.64 \\
\hline & Männlich & 2.54 & 2.14 & 1.77 \\
\hline \multirow[t]{3}{*}{ F-Werte } & & $\mathrm{F}_{\mathrm{BG}}=4.25^{*}$ & $\mathrm{~F}_{\mathrm{BG}}=38.63 * * *$ & $\mathrm{~F}_{\mathrm{BG}}=53.16^{* * *}$ \\
\hline & & $\mathrm{F}_{\mathrm{LG}}=2.46$ & $\mathrm{~F}_{\mathrm{LG}}=14.39 * * *$ & $\mathrm{~F}_{\mathrm{LG}}=9.56^{* *}$ \\
\hline & & $\mathrm{F}_{\mathrm{I}}=6.09 *$ & $\mathrm{~F}_{\mathrm{I}=0.14}$ & $F_{I=} 0.08$ \\
\hline
\end{tabular}

Anmerkung. $\mathrm{F}_{\mathrm{BG}}=\mathrm{F}-$ Wert Befragtengeschlecht, $\mathrm{F}_{\mathrm{LG}}=\mathrm{F}$-Wert Lehrergeschlecht, $\mathrm{F}_{\mathrm{I}}=\mathrm{F}$-Wert Interaktion. $* p<.05, * * p<.01, * * * p<.001$.

Auch für die Bewertung der Klassenlehrkraft ergibt sich kein Interaktionseffekt (Tabelle 2). Es ist also nicht der Fall, dass sich Jungen mit männlichen Lehrkräften stärker identifizieren (und auf dieser Basis besser bewerten) als mit weiblichen Lehrkräften. Es zeigt sich hingegen erstens, dass Mädchen allen Lehrkräften eine bessere Bewertung zukommen lassen als Jungen und dass zweitens weibliche Lehrkräfte bessere Noten erhalten - von Jungen wie von Mädchen.

Dieses Ergebnis bestätigt sich weitestgehend, wenn die einzelnen Dimensionen der Klassenlehrkraftbewertung betrachtet werden. Die Gerechtigkeit und Nettigkeit wird von den Mädchen besser eingeschätzt als von den Jungen; und die Klassenlehrerinnen erhalten auf beiden Dimensionen von allen Schülern bessere Noten. Nur hinsichtlich der Spannungseinschätzung ergibt sich eine signifikante Interaktion: Grundschülerinnen bewerten den Unterricht von Klassenlehrerinnen als spannender als den Unterricht von Klassenlehrern. Jungen machen hingegen keinen Unterschied, je nachdem, ob sie von einer Klassenlehrerin oder einem Klassenlehrer unterrichtet werden.

Grundsätzlich lässt sich folgern, dass das Geschlecht der Klassenlehrkraft keine geschlechtsspezifischen Folgen auf die Noten oder die Bewertung hat. In Tabelle 3 und Tabelle 4 wird der Frage nachgegangen, ob dieser Befund möglicherweise für ausgewählte Gruppen an Kindern gelten könnte. Hierfür werden einerseits die Migranten, andererseits die durch schulisches Gewaltverhalten auffälligen Kinder betrachtet. Möglicherweise haben es männliche Migranten bzw. männliche Schulgewalttäter bei 
weiblichen Lehrkräften schwerer, gute Noten zu erzielen als bei männlichen Lehrkräften.

Die Herkunft der Kinder wurde über die Fragen bestimmt, aus welchem Land die leiblichen Eltern stammen und in welchem Land die Kinder selbst geboren wurden (vgl. Baier et al., 2010, S. 261f). Mehr als jeder vierte Befragte hat einen Migrationshintergrund (27.6\%), wobei die größten Migrantengruppen durch Kinder mit türkischem Hintergrund bzw. durch Kinder aus Ländern der ehem. Sowjetunion gestellt werden (6.5 bzw. 5.2\%). Wie die Ergebnisse aus Tabelle 3 belegen, wird erneut für keine der betrachteten Noten ein signifikanter Interaktionseffekt ausgegeben. Bezüglich der Mathematiknote zeigt sich, dass männliche Migranten signifikant bessere Noten erzielen (bei weiblichen wie bei männlichen Lehrkräften) und dass männliche Lehrkräfte schlechtere Noten erteilen als weibliche Lehrkräfte. Für die Deutschnote deutet sich erneut an, dass männliche Migranten bei männlichen Lehrkräften etwas bessere Noten erzielen (nicht signifikant). Von einer Benachteiligung männlicher Migranten durch weibliche Lehrkräfte kann damit keine Rede sein. Für die Bewertung der Klassenlehrkraft ergibt sich wie für die Gesamtstichprobe, dass weibliche Migranten bessere Bewertungen abgeben und dass weibliche Lehrkräfte bessere Noten erhalten.

Tabelle 3. Noten und Klassenlehrkraftbewertungen nach Geschlecht des Schülers und der Lehrkraft - nur Kinder mit Migrationshintergrund (Mittelwerte).

\begin{tabular}{|c|c|c|c|c|c|}
\hline $\begin{array}{l}\text { Geschlecht } \\
\text { Befragter }\end{array}$ & $\begin{array}{l}\text { Geschlecht } \\
\text { Lehrkraft }\end{array}$ & $\begin{array}{c}\text { Deutschnote } \\
(N=1,720)\end{array}$ & $\begin{array}{c}\text { Mathematik- } \\
\text { note } \\
(\mathrm{N}=\mathbf{1 , 6 7 7 )}\end{array}$ & $\begin{array}{l}\text { Sportnote } \\
(N=1,470)\end{array}$ & $\begin{array}{c}\text { Bewertung } \\
\text { Klassenlehr- } \\
\text { kraft } \\
(\mathbf{N}=\mathbf{2 , 1 0 0 )}\end{array}$ \\
\hline \multirow[t]{2}{*}{ Männlich } & Weiblich & 2.88 & 2.52 & 1.93 & 2.28 \\
\hline & Männlich & 2.73 & 2.76 & 2.03 & 2.48 \\
\hline \multirow[t]{2}{*}{ Weiblich } & Weiblich & 2.72 & 2.86 & 2.07 & 2.00 \\
\hline & Männlich & 2.76 & 3.02 & 2.06 & 2.19 \\
\hline \multirow[t]{3}{*}{ F-Werte } & & $\mathrm{F}_{\mathrm{BG}}=0.31$ & $\mathrm{~F}_{\mathrm{BG}}=14.82^{* * *}$ & $\mathrm{~F}_{\mathrm{BG}}=2.54$ & $\mathrm{~F}_{\mathrm{BG}}=14.08^{* * *}$ \\
\hline & & $\mathrm{F}_{\mathrm{LG}}=0.23$ & $\mathrm{~F}_{\mathrm{LG}}=6.43^{*}$ & $F_{L G}=0.72$ & $\mathrm{~F}_{\mathrm{LG}}=6.58^{*}$ \\
\hline & & $\mathrm{F}_{\mathrm{I}}=0.66$ & $\mathrm{~F}_{\mathrm{I}=0.25}$ & $\mathrm{~F}_{\mathrm{I}=0.91}$ & $\mathrm{~F}_{\mathrm{I}=0.00}$ \\
\hline
\end{tabular}

Anmerkung. $\mathrm{F}_{\mathrm{BG}}=\mathrm{F}-$ Wert Befragtengeschlecht, $\mathrm{F}_{\mathrm{LG}}=\mathrm{F}-$ Wert Lehrergeschlecht, $\mathrm{F}_{\mathrm{I}}=\mathrm{F}$-Wert Interaktion. ${ }^{*} p<.05, * * p<.01, * * * p<.001$. 
Tabelle 4. Noten nach Geschlecht des Schülers und der Lehrkraft - nur Kinder, die in der Schule Gewaltverhalten gezeigt haben (Mittelwerte).

\begin{tabular}{|c|c|c|c|}
\hline $\begin{array}{l}\text { Geschlecht Befrag- } \\
\text { ter }\end{array}$ & $\begin{array}{l}\text { Geschlecht Lehr- } \\
\text { kraft }\end{array}$ & $\begin{array}{l}\text { Mathematiknote } \\
\qquad(N=792)\end{array}$ & $\begin{array}{l}\text { Sportnote } \\
(\mathrm{N}=692) \\
\end{array}$ \\
\hline \multirow[t]{2}{*}{ Männlich } & Weiblich & 2.52 & 1.93 \\
\hline & Männlich & 2.60 & 2.11 \\
\hline \multirow[t]{2}{*}{ Weiblich } & Weiblich & 2.90 & 1.99 \\
\hline & Männlich & 2.95 & 2.00 \\
\hline \multirow[t]{3}{*}{ F-Werte } & & $\mathrm{F}_{\mathrm{BG}}=9.96 * *$ & $\mathrm{~F}_{\mathrm{BG}}=0.08$ \\
\hline & & $\mathrm{F}_{\mathrm{LG}}=0.32$ & $\mathrm{~F}_{\mathrm{LG}}=1.11$ \\
\hline & & $\mathrm{F}_{\mathrm{I}=0.02}$ & $\mathrm{~F}_{\mathrm{I}=0.97}$ \\
\hline
\end{tabular}

Anmerkung. $\mathrm{F}_{\mathrm{BG}}=\mathrm{F}-$ Wert Befragtengeschlecht, $\mathrm{F}_{\mathrm{LG}}=\mathrm{F}-$ Wert Lehrergeschlecht, $\mathrm{F}_{\mathrm{I}}=\mathrm{F}-$ Wert Interaktion. ${ }^{*} p<.05, * * p<.01, * * * p<.001$.

Werden die Auswertungen auf Kinder eingeschränkt, die in den letzten vier Wochen vor der Befragung durch schulische Gewalt in Erscheinung getreten sind, ergeben sich ebenso wenige Hinweise auf eine unterschiedliche Leistungsbewertung (Tabelle 4). Um das schulische Gewaltverhalten zu bestimmen, wurden die Kinder gefragt, ob sie einen anderen Schüler geschlagen oder getreten haben (nicht aus Spaß) bzw. ob sie Sachen eines anderen Schülers mit Absicht kaputtgemacht haben. Dies trifft auf 13.3\% der Kinder zu, für Jungen häufiger als für Mädchen (19.2 zu 7.4\%). Da die Anzahl an Kindern, die Gewaltverhalten gezeigt haben, relativ gering ist und zugleich auch die Anzahl an männlichen Lehrkräften, die Deutsch unterrichten, können Auswertungen zur Deutschnote nicht präsentiert werden (die Fallzahl für Gruppe bei männlichen Lehrkräften unterrichteten Mädchen bzw. Jungen liegt unter 20). Für die Mathematik- und die Sportnote ergeben sich aber keine Hinweise darauf, dass männliche Schulgewalttäter insbesondere von weiblichen Lehrkräften benachteiligt würden. Tendenziell erhalten sie von den männlichen Lehrkräften schlechtere Noten (nicht signifikant).

\subsection{Befunde einer Befragung von Schülern der 9. Jahrgangsstufe}

Ebenfalls in den Jahren 2007 und 2008 wurden Befragungen unter Jugendlichen der neunten Jahrgangsstufe durchgeführt (Baier, Pfeiffer, Simonson \& Rabold, 2009). Diese erfolgten in 61 Landkreisen bzw. kreisfreien Städten in insgesamt 2,131 Klassen unter 44,610 Schülern. Die 
Rücklaufquote betrug $62.1 \%$. Auch in dieser Befragung wurde nach den Schulnoten und dem Geschlecht der Lehrkraft in den Fächern Deutsch, Mathematik und Sport gefragt. Zusätzlich wurden zu diesen Lehrkräften drei Einschätzungen erbeten: 1. Wie spannend und lehrreich ist der Unterricht? 2. Wie gerecht ist die Lehrkraft dir gegenüber? 3. Wie sehr unterstützt dich die Lehrkraft, wenn du Probleme hast? Die Bewertungen wurden also nicht für die Klassenlehrkraft, sondern jeweils für die Fachlehrkraft erfragt, wobei erneut entlang der Notenskala geantwortet werden sollte. Auch bei den Schülern der neunten Jahrgangsstufe korrelieren die einzelnen Bewertungen ausreichend hoch, um sie zu einer Skala zusammen zu fassen. Die Reliabilitäten betragen für die Deutschlehrkraft Cronbachs Alpha $=.81$, für die Mathematiklehrkraft .82 und die Sportlehrkraft .81. Die Korrelationen der Einschätzungen der verschiedenen Lehrkräfte korrelieren hingegen nur moderat miteinander $(r<.29)$, so dass davon auszugehen ist, dass die Jugendlichen in ihren Urteilen zwischen den einzelnen Lehrkräften differenzieren.

Das Problem, dass die Schüler einer Klasse z. T. abweichende Angaben zum Geschlecht der Lehrkraft gemacht haben, taucht bei Neuntklässlern noch deutlich häufiger auf als bei Viertklässlern, da aufgrund von Kurssystemen o. ä. die Schüler einer Klasse auch häufiger von verschiedenen Lehrkräften unterrichtet werden. Insbesondere im Sportunterricht werden in vielen Schulen Lehrkräfte unterschiedlichen Geschlechts eingesetzt. Deshalb haben wir auch hier entschieden, nur jene Klassen in die Auswertungen einzubeziehen, in denen von allen Schülern gleiche Angaben zum Lehrergeschlecht vorliegen. In die Analyse zur Deutschnote bzw. Deutschlehrkraftbewertung gehen deshalb nur Angaben von Schülern aus 1,237 Klassen, in die Analyse zur Mathematik aus 1,160 Klassen und in die Analyse zum Sport aus 636 Klassen ein. Es ist davon auszugehen, dass die gewählte Einschränkung zur Folge hat, dass einige Schülergruppen systematisch aus den Analysen ausgeschlossen werden. So zeigt ein Vergleich der Schüler, die nicht in die Auswertungen zum Fach Deutsch eingehen, mit Schülern, die in diese Auswertungen eingehen, dass vor allem bei den Gesamtschülern weniger Befragte berücksichtigt werden. In Gesamtschulen werden aufgrund des Kurssystems also häufiger Schüler durch Lehrer eines unterschiedlichen Geschlechts unterrichtet.

Werden die Durchschnittsnoten differenziert nach dem Geschlecht der Lehrkraft sowie dem Geschlecht des Jugendlichen betrachtet, so ergibt sich das in Tabelle 5 dargestellte Bild. Für die Deutsch- und die Mathematiknote findet sich nur ein signifikanter Effekt des Geschlechts des Befragten: Jungen erhalten deutlich schlechtere Deutsch-, aber bessere Ma- 
thematiknoten. Das Geschlecht der Lehrkraft spielt für die Notenvergabe keine Rolle. Zudem ist es nicht der Fall, dass weibliche Lehrkräfte männliche Schüler anders bewerten als männliche Lehrkräfte. Die These, dass sich das Lehrergeschlecht auf die Notenvergabe auswirkt, wird also auch in der neunten Jahrgangsstufe nicht bestätigt. ${ }^{1}$ Für die Sportnote weichen die Befunde von diesem bekannten Schema ab: Erstens zeigt sich, dass Jungen bessere Sportnoten erhalten als Mädchen. Zweitens vergeben Sportlehrerinnen signifikant bessere Noten als Sportlehrer. Dies ist drittens aber nur darauf zurückzuführen, dass Sportlehrer bei weiblichen Schülern deutlich schlechtere Noten geben als Sportlehrerinnen. Bei der Sportnote ergibt sich damit ein Effekt, nach dem die Lehrer die Mädchen benachteiligen; die Jungen werden durch die Lehrerinnen hingegen nicht benachteiligt. Allerdings sollten die Auswertungen zur Sportnote aufgrund des Ausschlusses zahlreicher Klassen (in denen sowohl männliche als auch weibliche Sportlehrer unterrichten) vorsichtig interpretiert werden.

Tabelle 5. Noten nach Geschlecht des Schülers und der Lehrkraft (Mittelwerte).

\begin{tabular}{|c|c|c|c|c|}
\hline $\begin{array}{l}\text { Geschlecht } \\
\text { Befragter }\end{array}$ & $\begin{array}{l}\text { Geschlecht } \\
\text { Lehrkraft }\end{array}$ & $\begin{array}{l}\text { Deutschnote } \\
(\mathrm{N}=\mathbf{2 4 , 5 0 7 )}\end{array}$ & $\begin{array}{c}\text { Mathematiknote } \\
\qquad(\mathrm{N}=\mathbf{2 3}, \mathbf{2 3 3})\end{array}$ & $\begin{array}{c}\text { Sportnote } \\
(N=13,119)\end{array}$ \\
\hline \multirow[t]{2}{*}{ Männlich } & Weiblich & 3.22 & 3.07 & 2.03 \\
\hline & Männlich & 3.21 & 3.02 & 2.02 \\
\hline \multirow[t]{2}{*}{ Weiblich } & Weiblich & 2.81 & 3.16 & 2.21 \\
\hline & Männlich & 2.83 & 3.16 & 2.41 \\
\hline \multirow[t]{3}{*}{ F-Werte } & - & $\mathrm{F}_{\mathrm{BG}}=1324.31 * * *$ & $\mathrm{~F}_{\mathrm{BG}}=63.82^{* * *}$ & $\mathrm{~F}_{\mathrm{BG}}=267.69 * * * *$ \\
\hline & & $\mathrm{F}_{\mathrm{LG}}=0.35$ & $\mathrm{~F}_{\mathrm{LG}}=3.52$ & $\mathrm{~F}_{\mathrm{LG}}=27.65^{* * *}$ \\
\hline & & $\mathrm{F}_{\mathrm{I}}=1.39$ & $F_{I}=2.77$ & $\mathrm{~F}_{\mathrm{I}=35.05 * * *}$ \\
\hline
\end{tabular}

Anmerkung. $\mathrm{F}_{\mathrm{BG}}=\mathrm{F}-$ Wert Befragtengeschlecht, $\mathrm{F}_{\mathrm{LG}}=\mathrm{F}-$ Wert Lehrergeschlecht, $\mathrm{F}_{\mathrm{I}}=\mathrm{F}-$ Wert Interaktion. ${ }^{*} p<.05, * * p<.01, * * * p<.001$.

Für die Auswertungen zur Bewertung der Lehrkräfte ergeben sich durchweg signifikante Befunde für die Interaktion aus Befragten- und Lehrergeschlecht (Tabelle 6). Anhand der Mittelwerte wird dabei deutlich, dass die

1 Der Befund, dass männliche Jugendliche von Lehrerinnen und Lehrern gleich benotet werden, bleibt erhalten, wenn in den Auswertungen die besuchte Schulform berücksichtigt wird. 
Schüler die Lehrer desselben Geschlechts besser beurteilen. Weibliche Schüler bewerten die Deutsch- und Mathematiklehrkraft dann am besten, wenn es sich um eine Lehrerin handelt; Jungen hingegen bewerten die männlichen Lehrkräfte besser. Sehr deutlich ist dieser Unterschied bei der Sportlehrkraft: Männliche Sportlehrer erhalten von männlichen Schülern die mit Abstand beste Bewertung. Für Jugendliche ist demnach die Geschlechtszugehörigkeit des Lehrers weit bedeutsamer, wenn es darum geht, dessen Unterricht und dessen Gerechtigkeit und Hilfsbereitschaft zu bewerten. Mit zunehmendem Alter nimmt in dieser Hinsicht die Identifikation mit gleichgeschlechtlichen Lehrkräften zu, da sich für die Viertklässler noch keine entsprechenden Effekte gezeigt haben. Diese stärkere Sympathie für die gleichgeschlechtlichen Lehrkräfte wird aber nicht ,erwidert": Wie gesehen, ,revanchieren" sich die Lehrkräfte nicht mit besseren Noten bei Schülern des gleichen Geschlecht; sie sind in ihren Bewertungen unabhängig davon, wie die Schüler über sie denken. Dies widerlegt die These, dass eine höhere Identifikation mit der gleichgeschlechtlichen Lehrkraft zu einer höheren Lernmotivation führt und darüber zu besseren Schulleistungen.

Tabelle 6. Lehrkraftbewertungen nach Geschlecht des Schülers und der Lehrkraft (Mittelwerte).

\begin{tabular}{|c|c|c|c|c|}
\hline $\begin{array}{l}\text { Geschlecht } \\
\text { Befragter }\end{array}$ & $\begin{array}{l}\text { Geschlecht } \\
\text { Lehrkraft }\end{array}$ & $\begin{array}{c}\text { Bewertung } \\
\text { Deutschlehrkraft } \\
(\mathrm{N}=\mathbf{2 4 , 5 8 4 )}\end{array}$ & $\begin{array}{c}\text { Bewertung Mathema- } \\
\text { tiklehrkraft } \\
(\mathrm{N}=\mathbf{2 3 , 3 0 9 )}\end{array}$ & $\begin{array}{c}\text { Bewertung } \\
\text { Sportlehrkraft } \\
(\mathrm{N}=\mathbf{1 3 , 1 8 5 )}\end{array}$ \\
\hline \multirow[t]{2}{*}{ Männlich } & Weiblich & 2.98 & 2.86 & 2.76 \\
\hline & Männlich & 2.89 & 2.83 & 2.46 \\
\hline \multirow[t]{2}{*}{ Weiblich } & Weiblich & 2.72 & 2.79 & 2.75 \\
\hline & Männlich & 2.86 & 2.94 & 2.82 \\
\hline \multirow[t]{3}{*}{ F-Werte } & & $\mathrm{F}_{\mathrm{BG}}=84.26 * * *$ & $\mathrm{~F}_{\mathrm{BG}}=1.53$ & $\mathrm{~F}_{\mathrm{BG}}=50.89 * * *$ \\
\hline & & $\mathrm{F}_{\mathrm{LG}}=1.99$ & $\mathrm{~F}_{\mathrm{LG}}=12.16^{* * *}$ & $\mathrm{~F}_{\mathrm{LG}}=22.64 * * *$ \\
\hline & & $\mathrm{F}_{\mathrm{I}}=55.40 * * *$ & $\mathrm{~F}_{\mathrm{I}}=29.62 * * *$ & $\mathrm{~F}_{\mathrm{I}=60.75 * * *}$ \\
\hline
\end{tabular}

Anmerkung. $\mathrm{F}_{\mathrm{BG}}=\mathrm{F}$-Wert Befragtengeschlecht, $\mathrm{F}_{\mathrm{LG}}=\mathrm{F}$-Wert Lehrergeschlecht, $\mathrm{F}_{\mathrm{I}}=\mathrm{F}-$ Wert Interaktion. $* p<.05, * * p<.01, * * * p<.001$.

\subsection{Auswertungen zu weiteren Einflussfaktoren der Schulleistungen}

Die These, dass das Geschlecht der Lehrkraft für die Schulleistungen und den Schulerfolg entscheidend ist, kann durch die präsentierten Auswer- 
tungen widerlegt werden. Als weitere Hypothese wurde formuliert, dass das Freizeitverhalten, insbesondere der mit dem Geschlecht variierende Medienkonsum eine Erklärung des differenziellen Schulerfolgs darstellen könnte. Aus diesem Grund soll abschließend ein Modell zur Erklärung der Schulnoten präsentiert werden, dass der Frage des Einflusses des Medienkonsums auf die Schulleistungen nachgeht. Dabei soll sich auf die Schüler der vierten Jahrgangsstufe konzentriert werden, da die in dieser Jahrgangsstufe erzielten Schulleistungen entscheidend für die Laufbahnempfehlungen und letztlich die gesamte weitere Schullaufbahn sind. Grundlage der nachfolgenden Ausführungen ist deshalb die in den Jahren 2007 und 2008 durchgeführte Befragung unter 7,844 Grundschulkindern.

Der Medienkonsum wurde in dieser Befragung in folgender Weise erfasst:

- Dauer des Konsums: Die Kinder wurden gefragt, wie lange sie am Tag vor der Befragung Computer gespielt bzw. Fern und Video gesehen haben. Die Zeiten sollten in einem Stundenplan eingetragen werden, in dem im Halbstundentakt von $7 \mathrm{Uhr}$ bis $23 \mathrm{Uhr}$ Kreuze für jene Tageszeiten gesetzt werden konnten, in denen einer bestimmten Tätigkeit nachgegangen wurde. Wenn für eine Tätigkeit keine Kreuze gesetzt wurden, wird davon ausgegangen, dass die entsprechende Tätigkeit nicht ausgeführt wurde; entsprechend wurde die Beschäftigungszeit auf null gesetzt. Die Kinder gehen pro Tag 1 Stunde und 49 Minuten den verschiedenen Tätigkeiten nach, wobei etwa doppelt so lange Fern/Video gesehen wie Computer gespielt wird. Jungen beschäftigen sich deutlich häufiger als Mädchen mit dem Medienkonsum (2:08 zu 1:29 Stunden). Dieser Geschlechterunterschied zeigt sich für alle Medienaktivitäten; er fällt am deutlichsten bei den Computerspielen aus.

- Inhalt des Konsums: Gefragt wurde danach, ob die Kinder schon einmal Filme geschaut oder Computerspiele gespielt haben, die erst ab 16 oder 18 Jahren frei gegeben sind. Mindestens eines dieser Formate haben $49.2 \%$ der Kinder genutzt. Jungen berichten dies zu $62.7 \%$, Mädchen zu 35.9\%.

Die Auswertungen belegen bereits starke Geschlechterunterschiede in der Dauer und den Inhalten des Medienkonsums. Zum Teil finden sich diese 
auch bei anderen Faktoren, die in der Analyse berücksichtigt werden sollen und aufgrund der Datenbasis berücksichtig werden können²

- Schichtzugehörigkeit: Da Grundschulkinder meist keine gesicherte Aussage zum Bildungsstand, Einkommen oder Berufsstatus der Eltern machen können und da in die Befragung keine Eltern einbezogen worden sind, liegen nur sehr wenige Daten zur sozialstrukturellen Position der Familie vor. Als Indikator der Schichtzugehörigkeit wird der Indikator zur Anzahl an Büchern im Haushalt herangezogen. Es gaben $50.7 \%$ der Kinder an, dass sie etwa ein Regal (51 bis 100) Bücher oder weniger Bücher zu Hause haben; 49.3\% berichteten von mehr als 100 Büchern. Zwischen Jungen und Mädchen gibt es bzgl. dieses Indikators keine Unterschiede.

- Elterliche Unterstützung: Um das elterliche Engagement bzgl. des Schulerfolgs zu erfassen, wurden die Kinder gebeten, getrennt für Mutter und Vater einzuschätzen, wie häufig diese bei den Hausaufgaben helfen (von ,1 - nie“ bis ,5 - immer"). Aus beiden Angaben wurde der Maximalwert gebildet, d. h. wenn die Mutter immer hilft, der Vater aber nie, geht der Wert zur Mutter in die Auswertungen ein. Diese Variablenbildung erscheint sinnvoll, weil nicht entscheidend ist, ob beide Elternteile helfen, sondern dass geholfen wird. Der Mittelwert zu dieser Variable beträgt 4.42; der deutlichen Mehrheit der Kinder wird also geholfen. Mädchen wird signifikant häufiger geholfen als Jungen (4.49 zu 4.36).

- Traditionelle Geschlechtsrollen: Kinder, die sich an diesen Rollenvorstellungen orientieren, sollten den schulischen Erfolg als weniger wichtig erachten und deshalb auch geringere Schulleistungen erzielen. Um dies zu prüfen, wurden den Kindern drei Aussagen zur Bewertung vorgelegt, die sich an die Skala zu Gewalt legitimierenden Männlichkeitsnormen (Enzmann, Brettfeld \& Wetzels, 2004) anlehnen („1 stimmt nicht“, „, - stimmt genau“): „Ein Junge muss sich gegen Beleidigungen zur Wehr setzen, sonst ist er ein Schwächling“, „Der Vater soll der Chef der Familie sein und darf sich, wenn es sein muss, auch mit Gewalt durchsetzen“ und „Ein richtiger Mann ist stark und beschützt seine Familie“. Die Antworten zu diesen Aussagen korrelie-

2 Die Intelligenz oder Persönlichkeitsfaktoren können nicht in die Auswertungen einbezogen werden, weil sie in der Befragung nicht bzw. nicht umfassend erfasst wurden. 
ren moderat miteinander, was sich in einem relativ niedrigem Reliabilitätskoeffizienten niederschlägt (Cronbachs Alpha $=.39$ ). Dennoch sollen die Items zu einer Mittelwertsskala „Männlichkeitsnormen“ zusammengefasst werden, deren Mittelwert in der Gesamtstichprobe 1.98 beträgt, also deutlich unter dem theoretischen Mittelwert von 2.5 liegt. Es stimmt also nur einer Minderheit der Schüler diesen Aussagen zu, Jungen allerdings signifikant häufiger als Mädchen $(2.05 \mathrm{zu}$ 1.91).

- Schulbindung: Hier sollten die Schüler folgende Aussagen auf einer Skala von „, - stimmt nicht“ bis , 4 - stimmt genau“ bewerten: „Schule macht Spaß“, „Morgens freue ich mich auf die Schule“ und „Ich gehe gern zur Schule“. Die Skala lehnt sich an das Messinstrument von Rauer und Schuck (2003) an; die interne Konsistenz ist als sehr gut zu bezeichnen (Cronbachs Alpha =.90). Der Mittelwert für die Gesamtstichprobe beträgt 2.73; Mädchen sind signifikant stärker an die Schule gebunden als Jungen (2.87 zu 2.60).

- Zeit für Lesen: Als eine Form der lernnahen Freizeitbeschäftigung wird die Zeit einbezogen, die mit Lesen verbracht wird. Dabei wird, wie bei Ermittlung der Medienkonsumzeiten auf die Angaben zum gestrigen Tag zurückgegriffen. Die Kinder gaben an, dass sie im Durchschnitt 41 Minuten Bücher gelesen hätten; Mädchen beschäftigen sich signifikant länger mit dem Lesen als Jungen (44 zu 37 Minuten).

Zusätzlich werden die beiden bereits vorgestellten Variablen des Migrationshintergrundes sowie des schulischen Gewaltverhaltens in die Analysen einbezogen. Erklärt werden soll die durchschnittliche Schulleistung, die als Mittelwert aus der Deutsch- und der Mathematiknote gebildet wurde. In Tabelle 7 sind die Ergebnisse verschiedener linearer Regressionsanalysen dargestellt.

Modell I belegt, dass Jungen minimal schlechtere Schulleistungen (hohe Werte $=$ schlechte Noten) aufweisen als Mädchen (nicht signifikant) . Da oben berichtet wurde, dass Jungen schlechtere Deutsch-, aber bessere Mathematiknoten erzielen, verwundert es nicht, dass es beim Mitteln beider Noten keinen Geschlechterunterschied gibt. Gleichwohl bedeutet dieser Befund auch, dass es in der vorliegenden Studie letztlich keinen geschlechtsspezifischen Bildungserfolg gibt, der zu erklären wäre. In anderen Befragungen konnte dies auch unter Verwendung der Durchschnittsnoten sichtbar gemacht werden (Baier \& Pfeiffer, 2011). Besonders deutlich drückt sich der Unterschied im Schulerfolg allerdings in den 
Laufbahnempfehlungen aus, die uns leider nicht zur Verfügung stehen, da sie nicht erhoben worden sind. Die Modelle II und III dienen dann nicht der Erklärung des geschlechtsspezifischen Schulerfolgs, sondern der Erklärung der Schulleistungen im Allgemeinen.

Dabei zeigt Modell II, dass Migranten deutlich schlechtere Schulleistungen erzielen und dass Kinder aus niedrigeren Sozialschichten (wenig Bücher zu Hause) ebenfalls schlechtere Noten erhalten. Wenn Kinder durch ihre Eltern bei den Hausaufgaben unterstützt werden und wenn sie eine hohe Schulbindung aufweisen, dann steht dies mit besseren Noten in Beziehung. Kinder wiederum, die in der Schule Gewaltverhalten ausführen und die sich an Männlichkeitsnormen orientieren, weisen schlechtere Schulleistungen auf.

Tabelle 7. Einflussfaktoren der Schulleistungen (OLS-Regression; abgebildet: Beta).

\begin{tabular}{|c|c|c|c|c|c|}
\hline & Modell I & Modell II & Modell III & Jungen & Mädchen \\
\hline Geschlecht: männlich & .01 & $-.03 * *$ & $-.06 * * *$ & - & - \\
\hline Herkunft: Migrant & & $.15 * * *$ & $.14 * * *$ & $.10 * * *$ & $.18 * * *$ \\
\hline wenig Bücher zu Hause $(<100)$ & & $.22 * * *$ & $.21 * * *$ & $.20 * * *$ & $.21 * * *$ \\
\hline Eltern helfen bei Hausaufgaben & & $-.06 * * *$ & $-.05 * * *$ & $-.06 * * *$ & $-.05 * *$ \\
\hline Männlichkeitsnormen & & $.10^{* * *}$ & $.09 * * *$ & $.08 * * *$ & $.09 * * *$ \\
\hline Schulbindung & & $-.12 * * *$ & $-.10 * * *$ & $-.08 * * *$ & $-.11 * * *$ \\
\hline Schulgewalttäter & & $.07 * * *$ & $.05 * * *$ & $.05^{* *}$ & $.07 * * *$ \\
\hline Zeit gestern: Lesen & & & $-.05 * * *$ & $-.06 * * *$ & $-.04 * *$ \\
\hline Zeit gestern: Medienkonsum & & & $.03 *$ & $.03 *$ & .02 \\
\hline Konsum altersgefährdender Medien & & & $.10 * * *$ & $.09 * * *$ & $.09 * * *$ \\
\hline $\mathbf{R}^{2}$ & .000 & .140 & .151 & .128 & .180 \\
\hline $\mathbf{N}$ & 7,158 & 7,158 & 7,158 & 3,534 & 3,623 \\
\hline
\end{tabular}

Anmerkung. $* p<.05,{ }^{* *} p<.01,{ }^{* * *} p<.001$.

In Modell III werden zusätzlich die Freizeitbeschäftigungen berücksichtigt. Das Lesen steht mit besseren Noten in Zusammenhang, die Zeit für Medienkonsum, insbesondere aber der Konsum mit altersgefährdenden Inhalten mit schlechteren Schulnoten. Da in Tabelle 7 standardisierte Koeffizienten abgetragen sind, lässt sich die Stärke des Einflusses direkt vergleichen: Dementsprechend kann gesagt werden, dass der Konsum altersgefährdender Medien der viertstärkste Einflussfaktor ist. Dies bestätigt, 
dass der Medienkonsum als ein relevanter Faktor für das Zustandekommen von Schulleistungen einzustufen ist. Dies gilt im Übrigen für Mädchen wie für Jungen. Die Modelle für die beiden Geschlechter zeigen, dass für alle betrachteten Faktoren vergleichbare Beziehungen $\mathrm{zu}$ beobachten sind. Auffällig ist nur, dass der Migrationshintergrund bei Mädchen die Schulleistungen stärker negativ beeinflusst als der Migrationshintergrund bei Jungen.

Wie das Modell III zuletzt zeigt, kehrt sich der Effekt des Geschlechts nach Kontrolle verschiedener Faktoren ins Negative um. Dies bedeutet, dass Jungen unter vergleichbaren Umständen sogar signifikant bessere Schulleistungen erzielen. Der Medienkonsum ist für das „Underachievement" der Jungen von besonderer Relevanz, wie der Vergleich der Modelle II und III sowie hier nicht abgebildete Analysen belegen. Allein die Berücksichtigung des Konsums altersgefährdender Medien verändert den standardisierten Koeffizienten von .01 auf -.04. Oder anders ausgedrückt: Fällt der Unterschied zwischen Jungen und Mädchen hinsichtlich der Durchschnittsnote in der Gesamtstichprobe noch zugunsten der Mädchen aus (2.48 zu 2.46), so kehrt sich der Geschlechterunterschied um, wenn nur jene Kinder betrachtet werden, die keine altersgefährdenden Medien konsumiert haben (2.29 zu 2.34). Die Folgerung ist, dass Jungen mit Blick auf die Schulleistungen unter ihren Möglichkeiten bleiben, weil sie derzeit noch deutlich häufiger mit altersgefährdenden Medien in Kontakt kommen.

\section{Zusammenfassung}

Die präsentierten Auswertungen lassen sich wie folgt zusammenfassen:

1. Das Geschlecht der Lehrkraft ist für die Vergabe von Schulnoten weitestgehend irrelevant. Festgestellt werden konnte, dass männliche Mathematiklehrer an Grundschulen schlechtere Noten verteilen und dass dies ebenfalls für männliche Sportlehrer an weiterführenden Schulen gilt. Letzteres ist vor allem darauf zurückzuführen, dass Schülerinnen von männlichen Sportlehrern schlechtere Noten erhalten.

2. Männliche Schüler erhalten von männlichen Lehrkräften in keiner der betrachteten Fälle bessere Schulnoten als von weiblichen Lehrkräften. Weder an Grundschulen noch an weiterführenden Schulen sind solche Muster zu erkennen. Damit ist nicht zu erwarten, dass eine Zunahme des männlichen Lehreranteils zu besseren Schulleistungen führt. Wir 
konnten diese Zusammenhänge allerdings nur für die Schulnoten prüfen. Der bisherige Forschungsstand bestätigt aber auch für Laufbahnempfehlungen oder Kompetenzwerte, dass nicht erwartet werden kann, dass männliche Schüler von männlichen Lehrkräften profitieren.

3. Es gibt keine Hinweise darauf, dass männliche Kinder von weiblichen Lehrkräften in der Notengebung benachteiligt werden. Solche Prozesse lassen sich auch nicht in Bezug auf Kinder mit Migrationshintergrund oder im Schulkontext verhaltensauffällige Kinder feststellen. Weibliche Grundschullehrerinnen kommen also mit allen Kindern gleichermaßen gut zurecht.

4. Die Bewertung der Lehrkraft entlang verschiedener Dimensionen (Unterrichtsqualität, Fairness, Unterstützung bzw. Nettigkeit) belegt in erster Linie, dass Schülerinnen bessere Noten verteilen als Schüler. Weibliche Lehrkräfte erhalten zudem etwas bessere Noten, wobei die Befunde hier nicht einheitlich sind. In der neunten Jahrgangsstufe wird sichtbar, dass Lehrkräfte des gleichen Geschlechts besser benotet werden. Eine solche Favorisierung des eigenen Geschlechts deutet sich in der vierten Klasse nur für die Mädchen und nur für die Dimension der Unterrichtsqualität (Spannung) an. Auch wenn damit für die neunte Klasse belegt werden kann, dass sich Schüler mit der Lehrkraft des eigenen Geschlechts etwas stärker identifizieren, bleibt dies folgenlos für ihre Leistungen; $d$. h. die höhere Identifikation führt nicht zu einer verstärkten Lernmotivation bzw. die Lehrkräfte bleiben in ihren Noteneinstufungen von diesen Sympathiebekundungen der gleichgeschlechtlichen Schüler unbeeinflusst.

5. Die Schulleistungen werden durch verschiedene Faktoren beeinflusst. Die Schichtzugehörigkeit sowie der Migrationshintergrund erweisen sich in den Analysen als wichtigste Prädiktoren, wobei Faktoren wie die Intelligenz oder die Persönlichkeit in den Auswertungen nicht berücksichtigt werden konnten. Neben diesen beiden Faktoren steht der Medienkonsum, und hier vor allem der Konsum altersgefährdender Formate, mit schlechten Schulleistungen in einer engen Beziehung. Dies lässt sich theoretisch u. a. mittels der Inhalts- und Löschungshypothese begründen (vgl. Mößle, Kleimann \& Rehbein, 2007, S. 21ff). Die Inhaltshypothese nimmt an, dass der Konsum gewalthaltiger Inhalte negativ auf das Sozialverhalten wirkt. Der Gewaltmedienkonsum kann dann ,zu einem negativen Kreislauf von Schulversagen, einer Entfremdung von der Kultur des Erfolgs und den Zielen, die durch die Schule repräsentiert werden, führen“ (Mößle et al., 2007, S. 41). Die Löschungshypothese geht davon aus, dass die hohe emotionale Invol- 
viertheit beim Betrachten von Gewaltinhalten dazu führt, dass der Transfer von schulisch gelerntem Wissen ins Langzeitgedächtnis erschwert wird. Für den Konsum altersgefährdender Medien lässt sich ein deutlicher Geschlechterunterschied feststellen, nach dem Jungen weit häufiger mit entsprechenden Filmen und Computerspielen in Kontakt kommen. Mit den Daten lässt sich jedoch nicht aufzeigen, dass der Medienkonsum den differenziellen Schulerfolg erklären kann, da sich bei den Durchschnittsnoten der Fächer Deutsch und Mathematik letztlich keine signifikanten Geschlechterunterschiede finden ließen. Belegt werden konnte aber mit den Daten, dass der geschlechtsspezifische Medienkonsum zur Folge hat, dass Jungen derzeit noch häufiger hinter ihren Leistungsmöglichkeiten zurück bleiben. Wenn Jungen genauso selten wie Mädchen altersgefährdende Medien nutzen würden, hätten sie signifikant bessere Schulleistungen.

Die Analyse der Einflussfaktoren des Schulerfolgs ist mit den vorliegenden Analysen nicht abgeschlossen. Der Frage, warum männliche Jugendliche seltener den Übergang auf das Gymnasium schaffen, dann letztlich seltener das Abitur ablegen und insgesamt auch häufiger in ihrer Bildungslaufbahn scheitern (gemessen anhand des Schulabbruchs), bedarf weiterer Analysen, die genau diese Indikatoren des Schulerfolgs und nicht die Schulnoten, wie hier geschehen, in den Mittelpunkt der Analyse rücken. Bei diesen Analysen kann aber darauf verzichtet werden, weiter das Geschlecht der Lehrkräfte oder die Geschlechtszusammensetzung des Lehrerkollegiums einzubeziehen. Stattdessen erscheint es sinnvoll, noch stärker die Rolle des Freizeitverhaltens der (männlichen) Schüler zu beleuchten und der Frage nachzugehen, inwieweit der Medienkonsum, speziell das Computerspielen, das von Jungen in weit höherem Maße ausgeführt wird als von Mädchen, Einfluss auf den geschlechtsspezifischen Schulerfolg hat.

\section{Literatur}

Baier, D. (2008). Entwicklung der Jugenddelinquenz und ausgewählter Bedingungsfaktoren seit 1998 in den Städten Hannover, München, Stuttgart und Schwäbisch Gmünd (Forschungsbericht No. 104). Hannover: Kriminologisches Forschungsinstitut Niedersachsen.

Baier, D. \& Pfeiffer, C. (2011). Mediennutzung als Ursache der schlechteren Schulleistungen von Jungen. In Hadjar (Hrsg.), Geschlechtsspezifische Ungleichheiten (S. 261-284). Wiesbaden: VS Verlag. 
Baier, D., Pfeiffer, C., Rabold, S., Simonson, J. \& Kappes, C. (2010). Kinder und Jugendliche in Deutschland: Gewalterfahrungen, Integration, Medienkonsum. Zweiter Bericht zum gemeinsamen Forschungsprojekt des Bundesministerium des Innern und des KFN (Forschungsbericht No. 109). Hannover: Kriminologisches Forschungsinstitut Niedersachsen.

Baier, D., Pfeiffer, C., Simonson, J. \& Rabold, S. (2009). Jugendliche in Deutschland als Opfer und Täter von Gewalt. Erster Forschungsbericht zum gemeinsamen Forschungsprojekt des Bundesministerium des Innern und des KFN (Forschungsbericht No. 107). Hannover: Kriminologisches Forschungsinstitut Niedersachsen.

Baier, D., Pfeiffer, C., Windzio, M. \& Rabold, S. (2006). Schülerbefragung 2005: Gewalterfahrungen. Schulabsentismus und Medienkonsum von Kindern und Jugendlichen. Abschlussbericht über eine repräsentative Befragung von Schülerinnen und Schülern der 4. und 9. Jahrgangsstufe. Hannover: Kriminologisches Forschungsinstitut Niedersachsen. Verfügbar unter: http:/www.kfn.de/ Forschungsbereiche_und_Projekte/Schuelerbefragungen/Schuelerbefragung_2005.h tm.

Diefenbach, H. (2010). Jungen - die „,neuen“ Bildungsverlierer. In G. Quenzel \& K. Hurrelmann (Hrsg.), Bildungsverlierer: Neue Ungleichheiten (S. 245-271). Wiesbaden: VS Verlag.

Ennemoser, M. (2003). Effekte des Fernsehens im Vor- und Grundschulalter. Ursachen, Wirkmechanismen und differenzielle Effekte. Nervenheilkunde, 22, 443453.

Enzmann, D., Brettfeld, K. \& Wetzels, P. (2004). Männlichkeitsnormen und die Kultur der Ehre. In D. Oberwittler \& S. Karstedt (Hrsg.), Soziologie der Kriminalität (S. 240-263). Wiesbaden: VS Verlag für Sozialwissenschaften.

Hadjar, A. \& Lupatsch, J. (2011). Geschlechterunterschiede im Schulerfolg. Spielt die Lehrperson eine Rolle? Zeitschrift für Soziologie der Erziehung und Sozialisation, $31,79-94$.

Helbig, M. (2010). Sind Lehrerinnen für den geringeren Schulerfolg von Jungen verantwortlich? Kölner Zeitschrift für Soziologie und Sozialpsychologie, 62, 93112.

Helbig, M. (2010a). Geschlecht der Lehrer und Kompetenzentwicklung der Schüler. In G. Quenzel \& K. Hurrelmann (Hrsg.), Bildungsverlierer: Neue Ungleichheiten (S. 273-288). Wiesbaden: VS Verlag.

Mößle, T. (2012). dick, dumm, abhängig, gewalttätig? Problematische Mediennutzungsmuster und ihre Folgen im Kindesalter. Ergebnisse des Berliner Längsschnitt Medien. Baden Baden: Nomos Verlag.

Mößle, T., Kleimann, M. \& Rehbein, F. (2007). Bildschirmmedien im Alltag von Kindern und Jugendlichen: Problematische Mediennutzungsmuster und ihr Zusammenhang mit Schulleistungen und Aggressivität (1. Aufl. Bd. 33). BadenBaden: Nomos. 
Neugebauer, M. (2011). Werden Jungen von Lehrerinnen bei den Übergangsempfehlungen für das Gymnasium benachteiligt? Eine Analyse auf Basis der IGLU-Daten. In A. Hadjar (Hrsg.), Geschlechtsspezifische Ungleichheiten (S. 235260). Wiesbaden: VS Verlag.

Neugebauer, M., Helbig, M. \& Landmann, A. (2010). Can the Teacher's Gender Explain the 'Boy Crisis' in Educational Attainment? Mannheimer Zentrum für Europäische Sozialforschung: Arbeitspapiere, 133.

Quenzel, G. \& Hurrelmann, K. (2010). Geschlecht und Schulerfolg: Ein soziales Stratifikationsmuster kehrt sich um. KZfSS Kölner Zeitschrift für Soziologie und Sozialpsychologie, 62(1), 61-91.

Rauer, W. \& Schuck, K. D. (2003). FEESS 3-4. Fragebogen zur Erfassung emotionaler und sozialer Schulerfahrungen von Grundschulkindern dritter und vierter Klassen. Göttingen: Hogrefe.

Rehbein, F., Kleimann, M. \& Mößle, T. (2010). Prevalence and Risk Factors of Video Game Dependency in Adolescence: Results of a German Nationwide Survey. Cyberpsychology, Behavior, and Social Networking, 13(3), 269-277.

Ribeaud, D. \& Eisner, M. (2009). Entwicklung von Gewalterfahrungen Jugendlicher im Kanton Zürich. Oberentfelden: Sauerländer Verlage. 
